\title{
Distribución y abundancia de murciélagos en bosques con diferente grado de intervención en el Parque Nacional Natural Gorgona (Colombia)
}

\author{
Oscar Enrique Murillo-García ${ }^{1 *}$ \& María Juliana Bedoya-Durán ${ }^{1}$ \\ 1. Departamento de Biología. Universidad del Valle, Cali, Colombia; oscar.murillo@correounivalle.edu.co; \\ ecologia@univalle.edu.co
}

Recibido 18-X-2013. Corregido 20-XI-2013. Aceptado 19-XII-2013.

\begin{abstract}
Distribution and abundance of bat assemblages among different forest covers in Gorgona National Natural Park, Colombia. Bats are important for maintenance of ecological processes in tropical forests since they are among the most abundant mammals and play a crucial role in tropical succession. Due to the importance of bats to forest dynamics and to the lack of ecological information, a study was conducted to quantify the variation in species richness, abundance and occurrence of bats in forest with different levels of perturbation in Gorgona National Natural Park; accounting for imperfect detectability. Bats were captured with mist-nets in three areas that differed in their perturbation levels (2 sites per area). A total of 670 bats representing 10 species and three families (Emballonuridae, Phyllostomidae y Vespertilionidae) were captured. The frugivorous species Dermanura rosenbergi (44.1\%), Carollia brevicauda (20.9\%) and Artibeus lituratus (30.1\%) were the most captured species, and 6 out of 10 were insectivorous. Detection probability $(p)$ increased with the increase in perturbation level $\left(p_{\text {perturbed }}>p_{\text {secondary }}>p_{\text {primary }}\right)$, and was highest for frugivorous bats. The best model for occurrence $(\Psi)$ revealed that probabilities of occurrence increased with perturbation levels $\left(\Psi_{\text {perturbe }}\right.$ $\mathrm{d}>\Psi_{\text {secondary }}>\Psi_{\text {primary }}$ ) and differed among species. Substantial differences in abundance were due to an additive effect of perturbation level, body size, and guild. Abundance increased with perturbation level ( $N_{\text {perturbed }}>N_{\text {seco }}$ ${ }_{\text {ndary }}>N_{\text {primary }}$ ), decreased with body size, and was highest for frugivorous bats. Bats had higher occurrence and abundance values in most disturbed areas, but they also occurred in less disturbed areas. Consequently, bats can potentially eat fruits from different stages of succession, promoting seed movement among zones that are suitable for colonization. Bats should be considered a conservation target for Gorgona. Rev. Biol. Trop. 62 (Suppl. 1): 419-434. Epub 2014 February 01.
\end{abstract}

Key words: Detection probability, occurrence probability, guilds, body size, species richness.

El conocimiento de las causas y consecuencias de la variaciones en distribución y abundancia de organismos es crucial para la conservación de la diversidad biológica. Por lo tanto la evaluación de los efectos de la perturbación sobre la composición y abundancia de las comunidades biológicas provee información importante para las decisiones de conservación y las acciones de manejo (Lim \& Engstrom, 2001; Patterson, Willig \& Stevens, 2003), particularmente en áreas naturales protegidas. Para tales propósitos, es particularmente útil el estudio y monitoreo de indicadores biológicos adecuados (Mooney, Lubchenco, Dirzo \& Sala,
1995). Este es el caso de los murciélagos, los cuales son de gran importancia en los bosques neotropicales debido a su abundancia, diversidad y múltiples funciones ecológicas (Fenton et al., 1992; Brosset, Charles-Dominique, Cockle, Cosson \& Masson, 1996), criterios importantes para considerar un taxón como indicador biológico (Noss, 1990; Mooney et al., 1995).

Los murciélagos constituyen el orden más importante de mamíferos en los bosques neotropicales representando el $39 \%$ de las especies de mamíferos (Voss \& Emmons, 1996; Emmons \& Feer, 1997) y presentando una gran diversidad de hábitos alimenticios: insectívoros, 
frugívoros, nectarívoros, carnívoros y hematófagos (Tirira, 2008). Consecuentemente, los murciélagos son de gran importancia dentro de cualquier ecosistema, puesto que son controladores de poblaciones de insectos, y contribuyen con la regeneración de los bosques mediante los procesos de polinización y dispersión de semillas (Emmons \& Feer, 1997; Soriano, 2000; Muñoz, 2001; Cleveland et al., 2006). Se ha demostrado que los murciélagos pueden ser utilizados para evaluar el nivel de perturbación de un sitio, ofreciendo una visión funcional que es complementaria a lo que puede ser percibido con medidas de diversidad o estructura vegetal (Fenton et al., 1992; Medellín, Equihua \& Amin, 2000). Sin embargo, a pesar de las numerosas contribuciones realizadas por los animales, el estudio del cambio sucesional en ecosistemas tropicales se ha limitado a cambios en la composición y estructura de las comunidades de plantas.

La Isla Gorgona está cubierta por bosque húmedo tropical, uno de los ecosistemas más diversos del planeta, aunque sus diferentes áreas han sido sometidas a diferentes procesos históricos de perturbación antrópica. La isla presentó altos niveles de perturbación debido al funcionamiento de una prisión de alta seguridad durante parte de la segunda mitad del siglo XX (Torres, 1986). Esta intervención se debió al establecimiento de cultivos, tala de árboles y a la construcción de edificaciones. Durante este periodo, el paisaje se modificó drásticamente por la desaparición de cerca del $70 \%$ del bosque nativo (Torres, 1986). Posteriormente, después de la declaración de la isla como Parque Nacional Natural (PNN), a mediados de los años 80 , la cobertura vegetal se ha recuperado considerablemente, a diferentes niveles, a través de la isla generando un gradiente sucesional. Sin embargo, a pesar de la importancia de los murciélagos para la estabilidad de los ecosistemas, no se tiene un conocimiento profundo sobre sus patrones de distribución y abundancia en el PNN Gorgona.

El objetivo del presente estudio fue evaluar la variación de la comunidad de murciélagos a través del gradiente de perturbación antropogénico que se presenta en el PNN Gorgona. Para cumplir con el objetivo, se cuantificaron las tasas de presencia, la abundancia y la riqueza en tres diferentes tipos de cobertura, a través de modelos estadísticos que consideran que la detectabilidad de las especies es imperfecta (MacKenzie et al., 2006; Royle \& Dorazio, 2008). Hasta el presente, pocos estudios han evaluado los cambios en las comunidades de murciélagos en bosques tropicales durante el proceso de sucesión (Medellín et al., 2000; Clarke, Rostant \& Racey, 2005; Castro-Luna, Sosa \& Castillo-Campos, 2007; Willig et al., 2007; Avila-Cabadilla, Stoner, Henry \& Alvarez-Añorve, 2009) y ninguno ha utilizado modelos que consideran las diferencias temporales y espaciales en detectabilidad. Estudios previos han encontrado que la detección de especies de murciélagos tropicales es imperfecta y puede estar influenciada por el sitio, la temporada o el método de muestreo (Meyer et al., 2011). Por lo tanto, la separación de la presencia real frente a la ausencia aparente, es importante para realizar inferencia sobre atributos como presencia, riqueza y abundancia de animales, puesto que sí la detectabilidad es imperfecta los conteos tienden a subestimar su distribución y abundancia (Williams et al., 2002; MacKenzie et al., 2006).

\section{MATERIALES Y MÉTODOS}

Área de Estudio: El Parque Nacional Natural Gorgona (PNN Gorgona) es un área protegida que se localiza en el Océano Pacífico al suroccidente colombiano e incluye territorio insular y marino. El área terrestre de la Isla Gorgona es de 1333.29ha y presenta elevaciones entre 0 y 338m. (PNN Gorgona 2004). Actualmente, la mayor parte de la isla está cubierta por especies vegetales de crecimiento secundario y solo una porción, se ha mantenido cubierta por bosque nativo, debido a su difícil acceso (Acevedo, Beltrán \& Caicedo, 2004; Cuevas-Correa, 2007).

Trabajo de campo: La cobertura boscosa del PNN Gorgona se ha clasificado de acuerdo 
al grado de perturbación de la siguiente forma (Acevedo et al., 2004; Cuevas-Correa, 2007): (a) Bosque primario (Zona noroccidental que no ha sido intervenida debido principalmente a su difícil acceso); (b) Bosque secundario (Zona en regeneración natural que se ha recuperado después de la declaratoria como zona natural protegida) y (c) Bosque intervenido (Zona alrededor del poblado que incluye los senderos para el turismo en el borde suroriental de la isla). Consecuentemente, para determinar la distribución y abundancia de murciélagos, se seleccionaron dos unidades de muestreo en cada tipo de cobertura: Bosque primario: 1) Sendero hacia el Cerro Trinidad y 2) Sendero hacia el Mirador; Bosque secundario: 1) Sendero Playa Palmeras-Piedra Redonda y 2) Sendero Yundigua, y Zona intervenida: 1) Poblado y alrededores y 2) Sendero hacia la Azufrada (Fig. 1). Se realizaron tres eventos de muestreo (octubre 2010, marzo 2011 y mayo de 2011), en los que se realizaron capturas para cada tipo de cobertura. En cada noche se muestreó una unidad de muestreo empleando 10 redes de niebla, de $12 \mathrm{~m}$ de longitud ubicadas sobre transectos de 300-500m; las cuales se revisaron

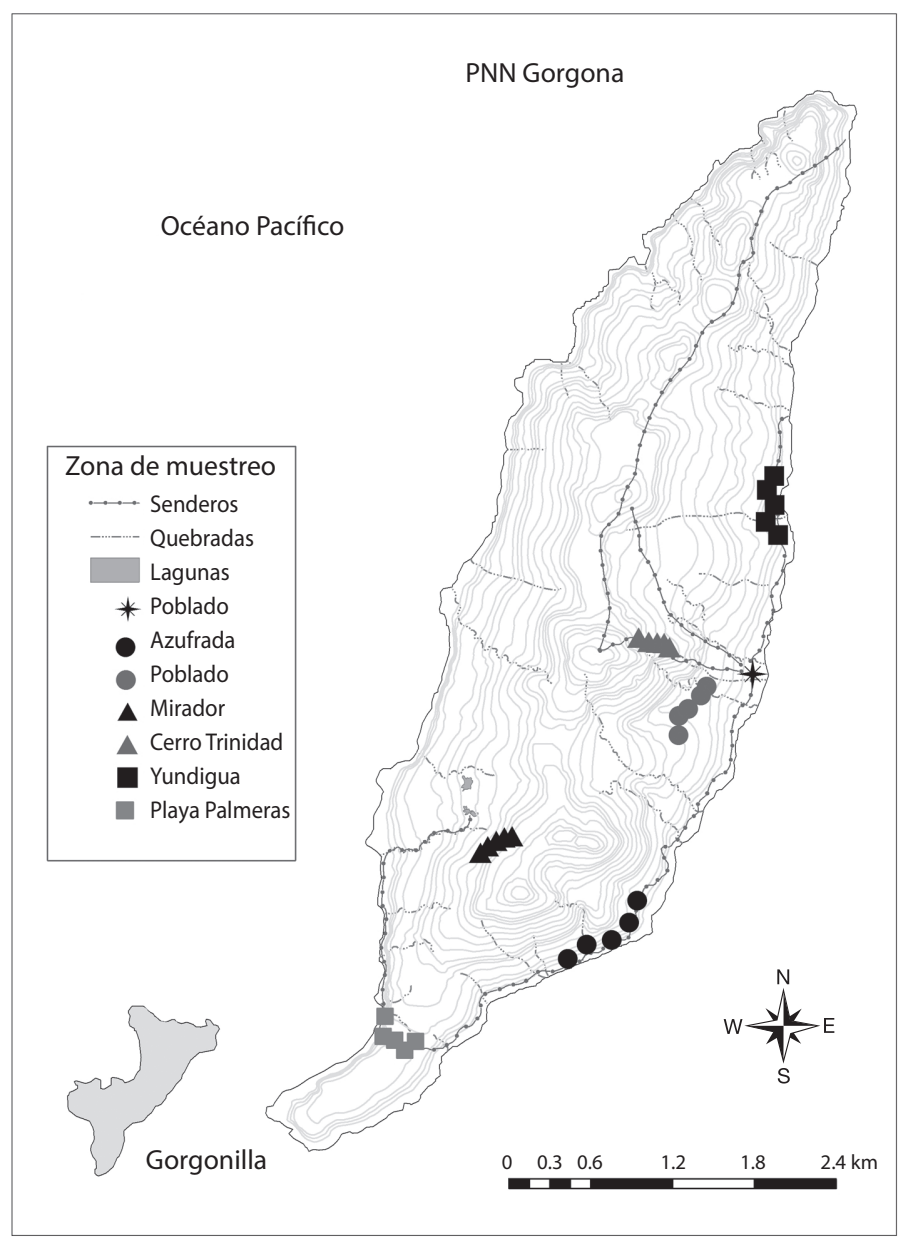

Fig. 1. Mapa del área de estudio con la ubicación de las unidades de muestreo utilizadas para el muestreo de la comunidad de murciélagos en el PNN Gorgona.

Fig. 1. Study area map showing the location of the sampling units used to sampling the bat assemblage in Gorgona National Natural Park. 
periódicamente entre las 17:30 y las 23:30h. En cada unidad de muestreo se capturaron murciélagos durante dos noches consecutivas, por lo cual se realizó un esfuerzo total de captura de 1080 horas-red durante 18 noches (seis noches por tipo de cobertura). Los murciélagos capturados se identificaron taxonómicamente y se marcaron, temporalmente, mediante perforaciones numéricas en el área del mesopatagio, con el fin de identificar individuos que fueran recapturados durante cada evento de muestreo.

Análisis de Datos: La inferencia acerca de la presencia y abundancia de animales puede ser errada cuando la probabilidad de detección no está incorporada en la estimación de estas variables (Williams et al., 2002; MacKenzie et al., 2006; Royle, Kéry, Gautier \& Schmid, 2007). Consecuentemente, con el fin de estimar la distribución espacial de la comunidad de murciélagos y evaluar los factores que la determinan, se estimó la presencia de especies en bosques con diferente grado de perturbación; utilizando un modelo de múltiples temporadas para múltiples especies (MacKenzie, Nichols \& Hines, 2003; Mackenzie, et al., 2006). De acuerdo con este, las tasas de presencia $(\psi)$ se definen como la probabilidad que una especie esté presente en un sitio, mientras la detectabilidad $(p)$ es la probabilidad que la especie sea detectada durante el muestreo en un sitio ocupado por esta (MacKenzie et al., 2002; 2006). Para la selección de los mejores modelos para las probabilidades de detección $(p)$ y presencia $(\psi)$ se siguió una aproximación secuencial, manteniendo las probabilidades de extinción $(\varepsilon)$ y de colonización $(\gamma)$ constantes debido a la condición de isla del área de estudio. En primer lugar, se identificó el mejor modelo para $p$ manteniendo la tasa de presencia constante y evaluando la influencia potencial de la especie, la clase de tamaño (pequeño, mediano, grande), el gremio trófico (insectívoro, nectarívoro y frugívoro), la categoría trófico-conductual (insectívoro de follaje, insectívoro aéreo, frugívoro sedentario, frugívoro nómada y nectarívoro), el tipo de cobertura (bosque intervenido, bosque secundario y bosque primario) y los efectos aditivos de estos factores sobre $p$. Posteriormente, se indentificó el modelo más parsimonioso para $\psi$ utilizando el mejor modelo para $p$ y evaluando la potencial influencia de los factores considerados sobre la probabilidad de presencia.

Con el fin de estimar la variación espacial en abundancia de la comunidad de murciélagos y evaluar los factores que la determinan, se estimó la abundancia de las especies en bosques con diferente grado de perturbación a través de modelos jerárquicos de abundancia que consideran las diferencias en detectabilidad (Royle \& Dorazio, 2006; Royle et al., 2007). Para la selección de los mejores modelos para las probabilidades de detección $(p)$ y la abundancia $(N)$ se siguió una aproximación secuencial, manteniendo las tasas de sobrevivencia $(\omega)$ y reclutamiento $(\gamma)$ constantes. En primer lugar, se identificó el mejor modelo para $p$ manteniendo la abundancia constante y evaluando la influencia potencial los factores considerados sobre $p$. Posteriormente, se determinó el modelo más parsimonioso para $N$ utilizando el mejor modelo para $p \mathrm{y}$, evaluando la potencial influencia de los factores considerados sobre la abundancia.

La inferencia se realizó a partir de múltiples modelos empleando una aproximación según la teoría de la información (Burnham \& Anderson, 2002), para lo cual se formularon hipótesis en forma de modelos probabilísticos con el fin de evaluar la importancia de diferentes factores sobre las tasas de presencia y la abundancia de las comunidades de murciélagos. La selección de los modelos y la inferencia estadística se realizó con base en el criterio de información de Akaike (Burnham \& Anderson, 2002; Burnham, 2004), específicamente se utilizaron el delta AIC y el peso del AIC para evaluar el soporte de los datos para cada uno de los modelos evaluados. Los modelos de presencia y abundancia se estimaron con el paquete estadístico Unmarked (Fiske \& Chandler, 2011), a través del programa $\mathrm{R}$ versión 2.13.1

(R Development Core Team, 2012).

Tradicionalmente, para la estimación de la riqueza de las comunidades se han utilizado 
estimadores basados en curvas de rarefacción o en estimadores paramétricos y no-paramétricos (Gotelli \& Colwell, 2001; Chao, Chazdon, Colwell \& Shen, 2006; Chao, Colwell, Lin \& Gotelli, 2009; Gotelli \& Colwell, 2011). Sin embargo, aunque los métodos más robustos (estimadores no-paramétricos) reducen el sesgo inherente a los conteos directos de especies, estos métodos subestiman sustancialmente la riqueza debido a su incapacidad para estimar adecuadamente el número de especies no detectadas (Gotelli \& Colwell, 2011). Consecuentemente, el número de especies no se puede estimar directamente sin error, pues está influenciada por las probabilidades de detección (Royle \& Dorazio, 2008). De tal forma que la riqueza y otros atributos de las comunidades solo se pueden estimar adecuadamente, utilizando modelos que explícitamente consideren la detección imperfecta de especies durante los muestreos (Royle \& Dorazio, 2008). Por esta razón, se estimó la riqueza como la sumatoria de las probabilidades de presencia de todas las especies en cada tipo de bosque (MacKenzie et al., 2006):

$$
S_{j}=\sum_{i=1}^{S} \Psi_{i j}
$$

donde $S_{j}$ es la riqueza de especies en el hábitat $j, \psi_{i j}$ es la probabilidad de presencia de la especie $i$ en el hábitat $j$ y $S$ es el número total de especies capturadas durante el muestreo. La probabilidad de presencia de cada una de las especies se calculó a través del promedio de los modelos, (MacKenzie et al., 2006). Con el fin de evaluar si existen diferencias en la riqueza de especies, se compararon los intervalos de confianza $(95 \%)$ para el número esperado de especies entre los diferentes grados de intervención.

\section{RESULTADOS}

En total se capturaron 670 individuos, pertenecientes a 10 especies y tres familias: Emballonuridae, Phyllostomidae y Vespertilionidae (Cuadro 1). Los filostómidos constituyeron el grupo con mayor número de individuos y especies capturadas (siete especies), seguidos por los vespertiliónidos (dos especies) y emballonúridos (una especie). Las tres especies con mayor número de capturas fueron Dermanura ronsebergi (Thomas, 1897) con 196 individuos (44.1\% de las capturas), Artibeus lituratus (Olfers, 1818) con 134 individuos (30.2\%) y Carollia brevicauda (Schinz, 1821) con 93 individuos (20.9\%). Las otras especies estuvieron pobremente representadas en el muestreo con porcentajes entre $4.1 \%$ y $0.7 \%$ (Cuadro 1). Se presentó un mayor número de capturas en los bosques intervenidos (370) con respecto a los secundarios (164) y primarios (136). El gremio con el mayor número de individuos capturados correspondió a los frugívoros, para los tres tipos de bosque, seguido por los insectívoros y los nectarívoros (Cuadro 1). Por otra parte, el gremio trófico con el mayor número de especies capturadas correspondió a los insectívoros (6 especies), seguido por los frugívoros (3 especies) y los nectarívoros (1 especie) (Cuadro 1).

Distribución espacial de la comunidad de murciélagos: El mejor modelo para exlicar la variación en la probabilidad de detección $(p)$, incluyó el efecto del gremio trófico de las especies (Modelo 1, Cuadro 2A). Con base en este modelo, la probabilidad de detección ( \pm error estándar) fue diferente para los tres gremios encontrados: $p$ fue mayor para las especies frugívoras $(0.93 \pm 0.0373)$, intermedio para las nectarívoras $(0.60 \pm 0.0789)$ y bajo para las especies insectívoras $(0.15 \pm 0.0399)$. Adicionalmente, el modelo que incluyó un efecto aditivo del gremio trófico y el tipo de cobertura sobre $p$ (Modelo 2, Cuadro 2A) también obtuvo soporte por parte de los datos. Con base en este modelo, $p$ fue mayor en los bosques intervenidos para los tres gremios (frugívoros: $0.95 \pm 0.0310$, nectarívoros: $0.68 \pm 0.0951$, insectívoros: $0.19 \pm 0.0599)$, intermedia en los bosques secundarios (frugívoros: $0.93 \pm 0.0373$, nectarívoros: $0.58 \pm 0.0785$, insectívoros: $0.14 \pm 0.0379)$ y baja en los bosques primarios (frugívoros: $0.89 \pm 0.0581$, nectarívoros: 
CUADRO 1

Especies, gremio trófico, categoría trófica-conductual y número de individuos de murciélagos capturados en bosques con diferente tipo de cobertura en el PNN Gorgona

TABLE 1

Species, trophic guild, trophic-behavioral category and total number of bats captured in forest with different forest covert type in Gorgona Island National Park

\begin{tabular}{|c|c|c|c|c|}
\hline \multirow[b]{2}{*}{ Taxón } & \multirow{2}{*}{$\begin{array}{c}\text { Gremio y } \\
\text { Categoría } \\
\text { Trófica* }\end{array}$} & \multicolumn{3}{|c|}{ Abundancia por tipo de cobertura } \\
\hline & & $\begin{array}{c}\text { Zona } \\
\text { intervenida }\end{array}$ & $\begin{array}{c}\text { Bosque } \\
\text { Secundario }\end{array}$ & $\begin{array}{l}\text { Bosque } \\
\text { primario }\end{array}$ \\
\hline \multicolumn{5}{|l|}{ Emballonuridae } \\
\hline Saccopteryx leptura (Schreber, 1774) & I/IA & 4 & 1 & 0 \\
\hline \multicolumn{5}{|l|}{ Phillostomidae } \\
\hline Micronycteris megalotis (Gray, 1842) & $\mathrm{I} / \mathrm{IF}$ & 7 & 1 & 2 \\
\hline Mimon crenulatum (É. Geoffroy St.-Hilaire, 1803) & $\mathrm{I} / \mathrm{IF}$ & 0 & 0 & 1 \\
\hline Tonatia saurophila (Koopman \& Williams, 1951) & $\mathrm{I} / \mathrm{IF}$ & 4 & 4 & 0 \\
\hline Carollia brevicauda (Schinz, 1821) & $\mathrm{F} / \mathrm{FS}$ & 121 & 18 & 26 \\
\hline Choeroniscus minor (W. Peters, 1868) & $\mathrm{N} / \mathrm{N}$ & 4 & 0 & 1 \\
\hline Artibeus lituratus (Olfers, 1818) & $\mathrm{F} / \mathrm{FN}$ & 79 & 64 & 48 \\
\hline Dermanura rosenbergi (Thomas, 1897) & $\mathrm{F} / \mathrm{FN}$ & 146 & 75 & 58 \\
\hline \multicolumn{5}{|l|}{ Vespertilionidae } \\
\hline Eptesicus chiriquinus (O. Thomas, 1920) & $\mathrm{I} / \mathrm{IA}$ & 1 & 0 & 0 \\
\hline Myotis nigricans (Schinz, 1821) & I/IA & 4 & 1 & 0 \\
\hline
\end{tabular}

* I: Insectívoro (IF: Ins. de follaje y IA: Ins. Aéreo), F: frugívoro (FS: Frugívoro sedentario y FN: Frugívoro nómada) y N: nectarívoro.

* I: Insectivorous (IF: Canopy Insectivorous y IA: Aerial Insectivorous), F: frugivorous (FS: sedentary frugivorous y FN: Nomadic Frugivorous) and N: nectarivorous.

4.58 \pm 0.1153 , insectívoros: $0.09 \pm 0.0430$ ). No se encontró evidencia para un efecto de la especie, el tamaño corporal o las categorías trófico-conductuales sobre $p$ (Cuadro 2A). Para la modelación de las tasas de presencia de los murciélagos de Gorgona se consideró el modelo más parsimonioso para las probabilidades de detección (Modelo 1, Cuadro 2A).

El modelo que incluyó un efecto aditivo del tipo de hábitat y la especie fue el mejor para predecir la variación en las tasas de presencia (Modelo 1, Cuadro 2B). De acuerdo con este, la probabilidad de presencia de la comunidad de murciélagos tiende a ser mayor en hábitats más intervenidos, que en los de menor intervención, y se presentan diferencia entre las especies en cuanto a las tasas de presencia (Fig. 2). Adicionalmente, no se encontró evidencia del efecto del gremio trófico, la categoría trófica- conductual o del tamaño sobre la probabilidad de presencia de la comunidad de murciélagos de Gorgona (Cuadro 2B).

Variación espacial en abundancia de las especies: El mejor modelo para predecir la probabilidad de detección $(p)$ de la comunidad de murciélagos incluyó un efecto aditivo del tipo de cobertura y del gremio trófico (Modelo 1, Cuadro 3A). De acuerdo con este, $p$ aumentó hacia las zonas de bosque con mayor intervención y fue mayor para las especies frugívoras en los tres tipos de cobertura (bosque intervenido: $0.799 \pm 0.0413$, bosque secundario: $0.726 \pm 0.0515$ y bosque primario: $0.639 \pm 0.0840)$ intermedia para las nectarívoras (bosque intervenido: $0.646 \pm 0.0478$ bosque secundario: $0.549 \pm 0.0454$ y bosque primario: $0.448 \pm 0.0734)$ y baja para las insectívoras (bosque intervenido:0.455 \pm 0.0494 , bosque secundario: $0.358 \pm 0.0316$ y bosque primario: 
CUADRO 2

Tabla de comparación para determinar los mejores modelos para (A) las probabilidades de detección (p) y

(B) las probabilidades de presencia $(\Psi)$ de la comunidad de murciélagos en el PNN Isla Gorgona

TABLE 2

Model comparison table to investigate the best base models for: (A) detection probabilities (p) and (B) occurrence probabilities ( $\Psi$ ) for the bat assemblage in Gorgona Island

\begin{tabular}{|c|c|c|c|c|c|}
\hline Modelo & & No. de parámetros & AIC & Delta AIC & Peso AIC \\
\hline & \multicolumn{5}{|c|}{ (A) Detección } \\
\hline 1 & $p(\mathrm{gt})$ & 5 & 150.23 & 0.00 & 0.390 \\
\hline 2 & $p(\mathrm{cob}+\mathrm{gt})$ & 6 & 150.45 & 0.21 & 0.350 \\
\hline 3 & $p(\operatorname{tam}+\mathrm{gt})$ & 6 & 152.20 & 1.96 & 0.150 \\
\hline 4 & $p(\mathrm{ctc})$ & 5 & 152.96 & 2.72 & 0.100 \\
\hline 5 & $p(\mathrm{sp})$ & 5 & 160.28 & 10.04 & 0.002 \\
\hline 6 & $p(\operatorname{tam})$ & 5 & 206.66 & 56.43 & 0.000 \\
\hline 7 & $p()$. & 4 & 208.10 & 57.87 & 0.000 \\
\hline \multirow[t]{2}{*}{8} & $p(\mathrm{cob})$ & 5 & 208.67 & 58.43 & 0.000 \\
\hline & \multicolumn{5}{|c|}{ (B) Presencia } \\
\hline 1 & $\Psi(\mathrm{cob}+\mathrm{esp})$ & 7 & 148.66 & 0.00 & 0.31 \\
\hline 2 & $\Psi()$. & 5 & 150.23 & 1.57 & 0.14 \\
\hline 3 & $\Psi(\mathrm{cob})$ & 6 & 150.38 & 1.72 & 0.13 \\
\hline 4 & $\Psi(\mathrm{cob}+\mathrm{gt})$ & 7 & 150.98 & 2.32 & 0.10 \\
\hline 5 & $\Psi(\mathrm{sp})$ & 6 & 151.10 & 2.44 & 0.09 \\
\hline 6 & $\Psi(\mathrm{gt})$ & 6 & 152.15 & 3.49 & 0.05 \\
\hline 7 & $\Psi(\operatorname{tam})$ & 6 & 152.18 & 3.52 & 0.05 \\
\hline 8 & $\Psi(\mathrm{ctc})$ & 6 & 152.21 & 3.55 & 0.05 \\
\hline 9 & $\Psi(\operatorname{tam}+\mathrm{cob})$ & 7 & 152.38 & 3.72 & 0.05 \\
\hline 10 & $\Psi(\operatorname{tam}+\mathrm{gt})$ & 7 & 153.83 & 5.17 & 0.02 \\
\hline
\end{tabular}

$p=$ Probabilidad de detección, ${ }^{\#} \Psi=$ probabilidad de presencia, $()=$. constante, $\mathrm{gt}=$ gremio trófico, $\mathrm{sp}=\mathrm{especie}$, tam=clase de tamaño, cob=tipo de cobertura y ctc=categoría trófico-conductual.

Para la evaluación del mejor modelo para la probabilidad de detección se mantuvieron constantes las probabilidades de presencia, extinción y colonización. Para la evaluación del mejor modelo para las probabilidad de presencia se utilizó el mejor modelo para la probabilidad de deteccción, $\mathrm{p}(\mathrm{gt})$, y se mantuvieron constantes las probabilidades de extinción y colonización.

$p=$ Detection probability, ${ }^{\#} \Psi=$ occurrence probability, $()=$. constant, ct=trophic guild, $\mathrm{sp}=$ species, tam=body size class, $\mathrm{cob}=$ forest cover type y $\mathrm{ctc}=$ behavioral-trophic category.

To investigate the best model structure for detection probabilities, probabilities of occurrence, extinction and colonization were fixed as constant. To investigate the best model for occurrence probabilities, the best model for detection probabilities was used, $\mathrm{p}(\mathrm{gt})$, and probabilities of extinction and colonization were fixed as constant.

$0.271 \pm 0.0494)$. No se encontró evidencia de un efecto de la categoría trófico-conductual, la especie o el tamaño sobre las probabilidades de detección (Cuadro 3A).

El modelo más parsimonioso para predecir la variación en abundancia incluyó el efecto aditivo del gremio trófico, el grado de intervención y el tamaño corporal (Modelo 1, Cuadro 3B). De acuerdo con este modelo, la abundancia de las especies fue mayor para las especies frugívoras que para la nectarívora y las insectívoras, incrementó al disminuir la cobertura vegetal y disminuyó al aumentar el tamaño corporal (Fig. 3). No se encontró evidencia de variación en abundancia relacionada con la categoría trófico-conductual o la especie (Cuadro 3).

Comparación de la riqueza de especies entre los hábitats: La riqueza ( \pm error 


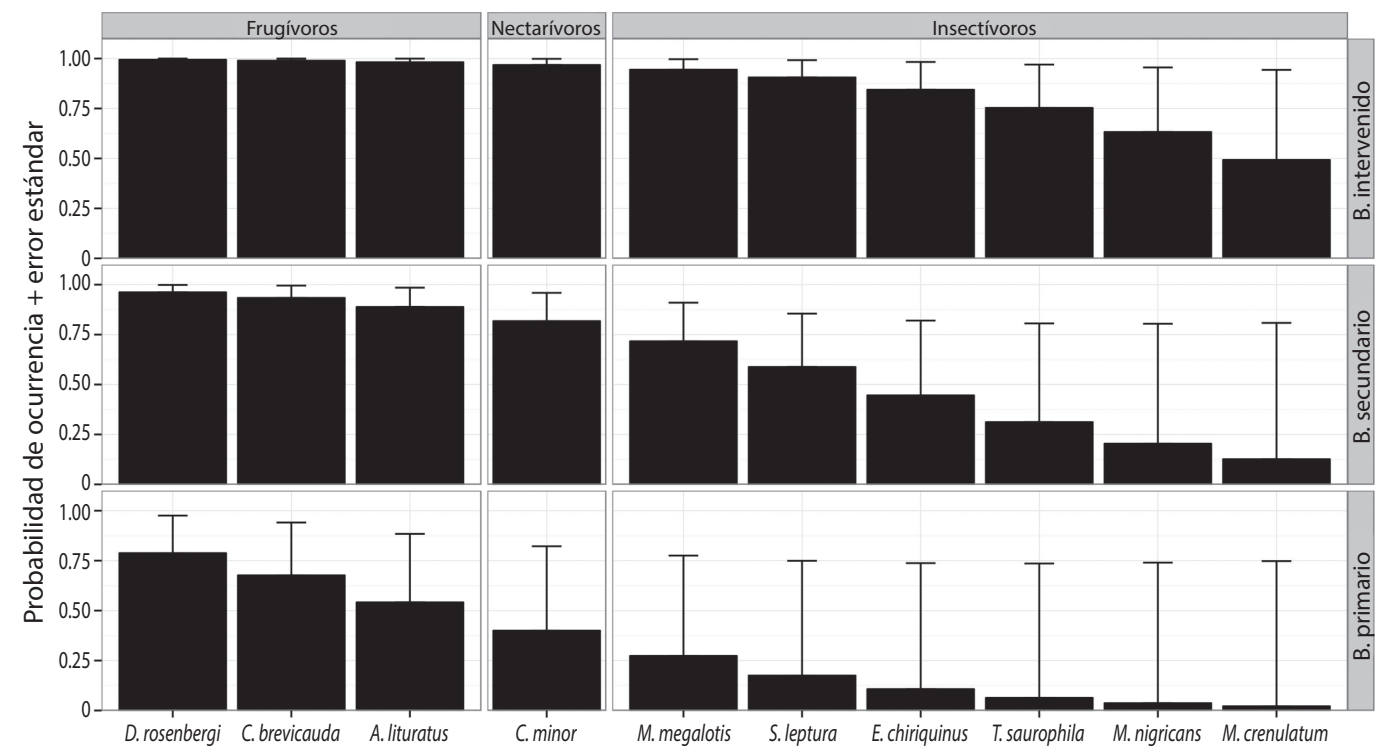

Fig. 2. Probabilidad de presencia ( \pm error estándar) de las especies de murciélagos en bosques con diferente grado de intervención.

Fig. 2. Occurrence probability ( \pm standard error) of bat species in forest with different perturbation levels.

estándar) en la zona intervenida fue de aproximadamente $8.32 \pm 0.73$ especies, mientras en la zona de bosque secundario fue de $6.87 \pm 1.21$ especies y en la zona de bosque primario fue de $4.91 \pm 1.95$ especies (Fig. 4). Sin embargo, la riqueza esperada de especies no es diferente entre los tipos de cobertura pues los intervalos de confianza se traslaparon considerablemente: bosque intervenido=[3.67-9.75], bosque secundario $=[3.19-9.24]$ y bosque primario $=[1.63-8.7]$.

\section{DISCUSIÓN}

Distribución espacial de la comunidad de murciélagos: Se encontró una variación considerable en la detectabilidad de las especies de murciélagos relacionada con diferencias entre gremios tróficos y tipos de cobertura. Las especies de murciélagos presentaron una mayor detectabilidad en las áreas de mayor intervención $\left(p_{\text {b.intervenido }}>p_{\text {b.secundario }}>p_{\text {b.primario }}\right)$ $\mathrm{y}$ esta fue siempre mayor para frugívoros que para los otros gremios ( $p_{\text {frugivoros }}>p_{\text {nectarivoros }}>p$ insectivoros). Por lo tanto al no considerar estas variaciones en la capacidad de detección de los murciélagos, se pudo haber subestimado las tasas de presencia de las especies y en las coberturas que presentaron menor detectabilidad con respecto a las de mayor detectabilidad. Esto pudo generar conclusiones erradas con respecto a los patrones de distribución y abundancia de la comunidad de especies (MacKenzie et al., 2002; 2006; Williams et al., 2002; Royle \& Dorazio, 2006; 2008). Al controlar por las diferencias en detección entre los gremios y las coberturas, las probabilidades de presencia de la comunidad dependieron del efecto combinado del tipo de cobertura y de las especies de murciélagos. Las tasas de presencia fueron diferentes entre las especies y fueron mayores en las zonas más intervenidas ( $\Psi_{\mathrm{b} \text {.intervenido }}>\Psi_{\mathrm{b}}$. secundario $>\Psi_{\text {b.primario }}$ ) para todas las especies.

Aunque las flores y los frutos son frecuentemente conspicuos y fáciles de localizar, los estudios que han cuantificado la variación temporal y espacial en recursos sugieren que las densidades de flores y frutos son más 
CUADRO 3

Tabla de comparación para determinar los mejores modelos para: (A) las probabilidades de detección (p) y (B) la abundancia (N) de la comunidad de murciélagos en el PNN Isla Gorgona

TABLE 3

Model comparison table for abundance analyses to investigate the best base model for: (A) detection probability (p) and $(\mathrm{B})$ abundance $(\mathrm{N})$ for bat assemblage at Gorgona Island

\begin{tabular}{|c|c|c|c|c|c|}
\hline Modelo & & No. de parámetros & AIC & Delta AIC & Peso AIC \\
\hline & \multicolumn{5}{|c|}{ (A) Detección } \\
\hline 1 & $p(\mathrm{cob}+\mathrm{gt})$ & 6 & 885.33 & 0.00 & 1.00 \\
\hline 2 & $p(\operatorname{tam}+\mathrm{gt})$ & 6 & 975.64 & 90.32 & 0.00 \\
\hline 3 & $p(\mathrm{gt})$ & 5 & 978.57 & 93.24 & 0.00 \\
\hline 4 & $p(\mathrm{ctc})$ & 5 & 989.55 & 104.22 & 0.00 \\
\hline 5 & $p(\mathrm{sp})$ & 5 & 1051.03 & 165.71 & 0.00 \\
\hline 6 & $p(\mathrm{cob})$ & 5 & 1648.25 & 762.93 & 0.00 \\
\hline 7 & $p()$. & 4 & 1660.53 & 775.21 & 0.00 \\
\hline \multirow[t]{2}{*}{8} & $\mathrm{p}(\operatorname{tam})$ & 5 & 1662.49 & 777.16 & 0.00 \\
\hline & \multicolumn{5}{|c|}{ (B) Abundancia } \\
\hline 1 & $N(\operatorname{tam}+\mathrm{cob}+\mathrm{gt})$ & 9 & 829.88 & 0.00 & 0.99 \\
\hline 2 & $N(\mathrm{cob}+\mathrm{gt})$ & 8 & 838.59 & 8.71 & 0.01 \\
\hline 3 & $N(\operatorname{cob}+\mathrm{sp})$ & 8 & 897.09 & 67.21 & 0.00 \\
\hline 4 & $N(\operatorname{tam}+\mathrm{gt})$ & 8 & 913.32 & 83.44 & 0.00 \\
\hline 5 & $N(\mathrm{gt})$ & 7 & 926.50 & 96.62 & 0.00 \\
\hline 6 & $N(\mathrm{sp})$ & 7 & 980.14 & 150.26 & 0.00 \\
\hline 7 & $N(\mathrm{cob})$ & 7 & 1438.56 & 608.68 & 0.00 \\
\hline 8 & $N(\mathrm{cob}+\mathrm{tam})$ & 8 & 1440.56 & 610.68 & 0.00 \\
\hline 9 & $N()$. & 6 & 1526.02 & 696.14 & 0.00 \\
\hline 10 & $N(\operatorname{tam})$ & 7 & 1527.98 & 698.10 & 0.00 \\
\hline
\end{tabular}

$p=$ Probabilidad de detección, ${ }^{\#} \Psi=$ probabilidad de presencia, $()=$. constante, gt=gremio trófico, $\mathrm{sp}=\mathrm{especie}$, tam=clase de tamaño, cob=tipo de cobertura y ctc=categoría trófico-conductual.

Para la evaluación del mejor modelo para la probabilidad de detección se mantuvieron constantes la abundancia, y las probabilidades de sobrevivencia y de reclutamiento. Para la evaluación del mejor modelo para la abundancia se utilizó el mejor modelo para la probabilidad de detección, $\mathrm{p}(\mathrm{cob}+\mathrm{gt})$, y se mantuvieron constantes las probabilidades de sobrevivencia y de reclutamiento.

$p=$ Detection probability, ${ }^{*} \Psi=$ occurrence probability, $()=$. constant, $\mathrm{ct}=$ trophic guild, $\mathrm{sp}=\mathrm{species}$, tam=body size class, $\mathrm{cob}=$ forest cover type y $\mathrm{ctc}=$ behavioral-trophic category.

To investigate the best model structure for detection probabilities, abundance and probabilities of survival and recruitment were fixed as constant. To investigate the best model for occurrence probabilities, the best model for detection probabilities were used, $\mathrm{p}(\mathrm{cob}+\mathrm{gt})$, and probabilities of survival and recruitment were fixed as constant.

variables que las de insectos (Karr, 1976; Pyke, 1983; Martin \& Karr, 1986). Por lo cual los vertebrados frugívoros y nectarívoros al alimentarse de fuentes que despliegan una variación considerable, a diferentes escalas espaciales y temporales, invierten la mayor parte del tiempo buscando alimento. En estos organismos el seguimiento de la abundancia de recursos alimenticios explica la mayoría de los movimientos diarios y estacionales, los cuales generalmente involucran movimientos entre hábitats a lo largo de gradientes sucesionales (Karr, 1981; 1990; Flemming, 1992). Por lo tanto, los frugívoros y nectarívoros pueden cambiar de hábitats con mayor probabilidad que los insectívoros (Martin \& Karr, 1986; Flemming, 1992), y se esperaría que estos presentaran mayores tasas de presencia a nivel del 


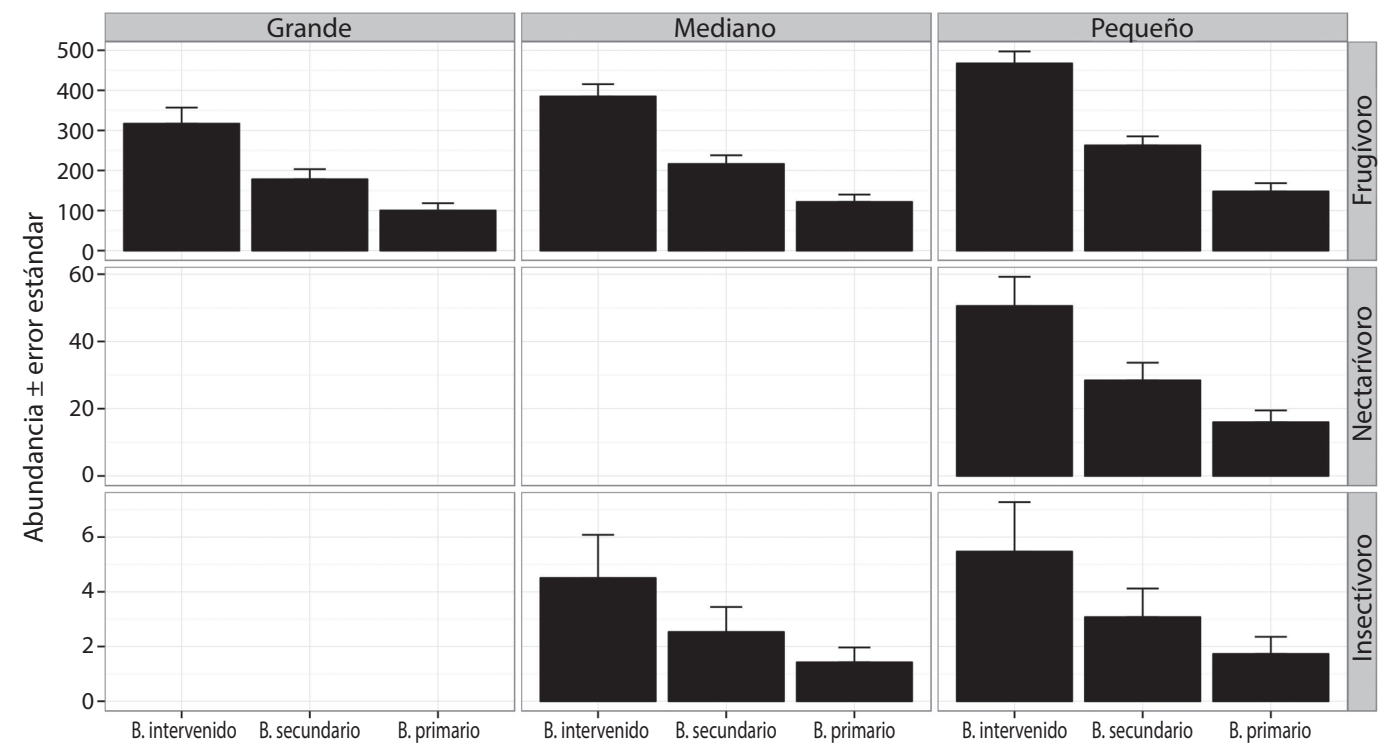

Fig. 3. Variación en abundancia (+ error estándar) de los gremios tróficos de las especies de murciélagos, por clases de tamaño en bosques con diferente grado de intervención.

Fig. 3. Variations in abundance (+ standard error) for trophic guilds of bats by size classes in forest with different perturbation levels.

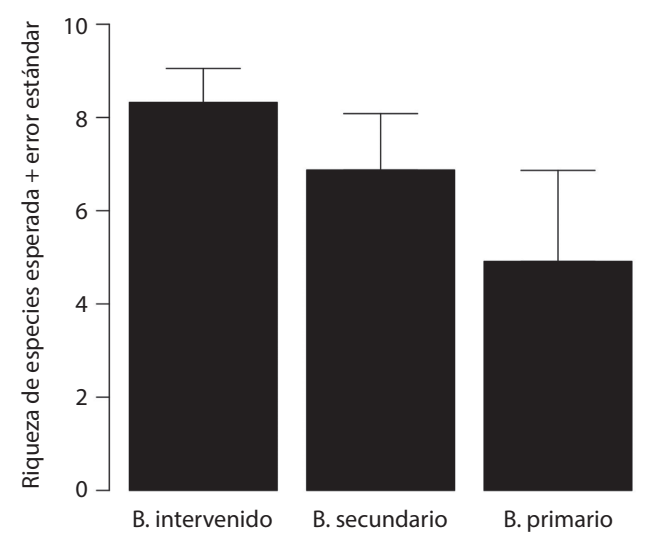

Fig. 4. Riqueza esperada ( + error estándar) de la comunidad de murciélagos en bosques con diferente grados de intervención.

Fig. 4. Predicted species richness (+ standard error) of bat assemblages in forests with different perturbation levels.

paisaje que los insectívoros. Sin embargo, no se encontró evidencia fuerte para una variación entre los gremios tróficos en las tasas de presencia con base en los datos obtenidos a partir del muestreo.
Abundancia de murciélagos: De igual forma que en los modelos de presencia, la variación en detectabilidad para los modelos de abundancia fue caracterizada por un efecto aditivo del gremio trófico y el tipo de cobertura del bosque. Por otra parte, las variaciones en la abundancia fueron descritas por un efecto combinado del gremio trófico, el tipo de cobertura, y el tamaño. De tal forma que la abundancia fue mayor para los frugívoros y nectarívoros que para los insectívoros, y las especies presentaron mayor abundancia en las zonas de mayor intervención $\left(N_{\mathrm{b} \text {. intervenido }}>N_{\mathrm{b} \text {.secundario }}>N_{\mathrm{b} \text { primario }}\right)$ y al disminuir el tamaño corporal. Los murciélagos frugívoros fueron los más abundantes en todas las zonas de la Isla. Estos presentaron una tendencia a ser más abundantes en las zonas de mayor perturbación dónde son comunes especies vegetales pioneras típicas de sucesiones tempranas, las cuales componen una importante proporción de la dieta de estos organismos (Hernández-Conrique, Iniguez-Davalos \& Storz, 1997; Estrada \& Coates-Estrada, 2001). La presencia de altas concentraciones de plantas de los géneros Piper, Solanum y Cecropia 
en los bordes y al interior de los bosques, así como a lo largo de quebradas y ríos, puede beneficiar a las especies del género Carollia (Galindo-González, 1998). Igualmente, la producción masiva de frutos de plantas del género Ficus atrae a las especies de los géneros Artibeus y Dermanura, que a su vez han desarrollado sincronía entre sus periodos reproductivos y las épocas de fructificación de estas especies vegetales (Galindo-González, 1998; Medellín et al., 2000; Estrada \& Coates-Estrada, 2001). Por otra parte, la mayor abundancia de insectívoros en las zonas intervenidas puede deberse a los procesos de urbanización en estas áreas debido a su cercanía al poblado. En esta zona se han generado cambios en la estructura de los recursos alimenticios debido a la reducción de la cobertura vegetal, a la introducción de especies de plantas ornamentales y a la presencia de fuentes de iluminación que aumentan y concentran el recurso de insectos (Rydell \& Baagøe, 1996; Longcore \& Rich, 2004).

El tamaño corporal es posiblemente una de las características más importantes de un organismo, pues está relacionada con muchas características biológicas, incluyendo la abundancia (Kerr \& Dickie, 2001; Brown, Gillooly, Allen, Savage \& West, 2004; Woodward et al., 2005). Uno de los patrones que se espera, a nivel de la comunidad, es la relación inversa entre abundancia y tamaño corporal de los organismos (White, Ernest, Kerkhoff \& Enquist, 2007). Este patrón fue soportado por los datos obtenidos en el muestreo, pues la mayor abundancia se presentó en la clase de tamaño pequeña para todos los grados de intervención de los bosques. Adicionalmente, debido a las altas densidades poblacionales que presentan las especies frugívoras y a la impredictabilidad de la disponibilidad de la fruta, se espera una alta competencia inter-específica dentro de este gremio (Flemming, 1992). Sin embargo, no se presenta un traslape en tamaño corporal entre las tres especies de frugívoros, lo cual puede indicar que utilizan recursos diferentes y de esta forma reducen la competencia y facilitan la coexistencia.
La mayor abundancia de especies frugívoras está de acuerdo a lo esperado en bosques tropicales, pero es notable la escasez de especies nectarívoras (una especie) y su baja abundancia. En condiciones naturales, la biomasa total de fruta en ecosistemas tropicales es mayor a la de otros recursos, como néctar e insectos, por lo cual las densidades de murciélagos frugívoros son mayores que las de nectarívoros y estas mayores que las de insectívoros (Flemming, 1992; Carrera, 2003). En la isla, contrario a lo esperado, se presentó una mayor abundancia esperada de insectívoros que de nectarívoros. Lo cual indica que la abundancia de nectarívoros está muy por debajo de lo esperado y debe ser una causa de preocupación debido a la importancia de este grupo para la estabilidad de los ecosistemas boscosos de la isla; especialmente si se considera que la isla se encuentra suficientemente alejada del continente como para que se presente eventos de recolonización.

Riqueza de especies de la comunidad de murciélagos: Los resultados sugieren que la riqueza de la comunidad de murciélagos es homogénea entre los bosques con diferente tipo de cobertura de la Isla. Por lo tanto, aunque no se registraron todas las especies en todos los tipos de bosque, es probable que estas compartan la mayoría de las especies. Sin embargo estas exhiben preferencias particulares por algún tipo de cobertura relacionadas con el gremio trófico. Siendo esto particularmente cierto para las especies insectívoras aéreas, las cuales presentaron mayor abundancia en la zona más intervenida y pueden ser difíciles de capturar al interior de los bosques debido a que éstos forrajean en áreas abiertas.

Conclusiones generales: A pesar de la importancia del sesgo inducido por los muestreos (Voss \& Emmons, 1996; Simmons \& Voss, 2001; Meyer et al., 2011), el problema de la detectabilidad no es considerado explícitamente en la gran mayoría de estudios de variación espacial en abundancia, presencia y riqueza de murciélagos (Medellín et al., 2000; Clarke et al., 2005; Castro-Luna et al., 2007; 
Willig et al., 2007; Avila-Cabadilla et al., 2009). Sin embargo, los resultados del presente estudio muestran que existen una considerable variación en detectabilidad relacionada con variaciones entre gremios tróficos y tipos de cobertura, lo cual está de acuerdo con previos estudios que han evaluado las fuentes de variación relacionadas con la detectabilidad de murciélagos tropicales (Meyer et al., 2011). Por lo tanto, considerar la detectabilidad es importante cuando se hace inferencia acerca de la presencia, la abundancia y la riqueza de especies animales porque los conteos observados están sesgados por la detección imperfecta y porque los ceros observados no necesariamente representan ausencia.

Nuestros resultados concuerdan con estudios previos que ha reportado que las comunidades de murciélagos despliegan fuertes respuestas a la pérdida de bosque y al cambio en el uso de la tierra (García-Morales, Badano \& Moreno, 2013; Medellín et al., 2000; Clarke et al., 2005; Castro-Luna et al., 2007; Willig et al., 2007; Avila-Cabadilla et al., 2009). Los resultados de las capturas también concuerdan con una serie de meta-análisis, en los cuales se comparó la frecuencia de presencia de especies de murciélagos entre bosques bién preservados y áreas utilizadas por humanos, y se encontró que en general las áreas utilizadas por humanos contienen más especies de murciélagos que los bosques bien preservados (GarcíaMorales et al., 2013). Sin embargo, la mayoría de los estudios analizados por García-Morales et al. (2013) no consideran las probabilidades de detección, por lo cual este patrón podría reflejar diferencias en detectabilidad más que diferencias en presencia sí las probabilidades de detección son consistentemente mayores en las áreas utilizadas por humanos que en bosques bien preservados; cómo se reporta en este trabajo. Previamente se ha demostrado que la detectabilidad a nivel de especies es usualmente imperfecta y que puede ser influenciada por un rango de factores externos como el sitio (Meyer et al., 2011), lo cual sugiere que los conteos directos pueden algunas veces estar sesgados y la inferencia derivada a partir de estos puede ser errada.

La relación directa entre la abundancia y el grado de perturbación no indíca que los bosques más intervenidos sean de mayor importancia para la aconservación. La mayoría de las especies de murciélagos de la isla son comunes de zonas intervenidas, por lo cual se podría esperar este patrón en la distribución y la abundancia de la comunidad. En la isla, aunque se presenta una preferencia de las especies de murciélagos por las áreas más intervenidas, los murciélagos también visitan las menos intervenidas. En este proceso las especies frugívoras se puede alimentar de frutos de especies pioneras y de estados sucesionales tardíos promoviendo la regeneración de los bosques tropicales al depositar las semillas en ambientes propicios para la colonización (Fleming \& Heithaus, 1981; Fleming \& Williams, 1990; Fenton et al., 1992; Gorchov, Cornejo, Ascorra \& Jaramillo, 1993; Law, Anderson \& Chidel, 1999; Schulze, Seavy \& Whitacre, 2000; Lobova, Mori, Blanchard, Peckham \& Charles-Dominique, 2003; Passos, Silva, Pedro \& Bonin, 2003; Loayza, Rios \& Larrea-Alcázar, 2006).

En el presente estudio se registraron seis especies de murciélagos insectívoros, siendo los más comunes Saccopteryx leptura (Schreber, 1774), Micronycteris megalotis (Gray, 1842), y Tonatia saurophila (Koopman \& Williams, 1951). Los murciélagos insectívoros pueden ser los principales consumidores de insectos, y dados los relativos grandes volúmenes consumidos (más del 100\% de la masa corporal por noche) y las largas distancias que atraviesan (varios kilómetros por noche), estos murciélagos juegan un papel importante en la disminución de las poblaciones de insectos nocturnos (Pierson, 1998; Cleveland et al., 2006). Además de la alta abundancia de especies frugívoras y del mayor número de especies insectívoras, es notable la presencia de tan solo una especie nectarívora, Choeroniscus minor (W. Peters, 1868), con una baja abundancia. Sin embargo, a pesar de su baja abundancia, $C$. minor es una especie clave por su función como polinizador al alimentarse principalmente de 
néctar y polen de especies vegetales típicas de bosques húmedos tropicales (Tirira, 2008). Por lo tanto, el estudio de la comunidad de murciélagos es de gran importancia para entender los procesos ecológicos que mantienen la estabilidad y generan la recuperación de los bosques, y para monitorear el estado de recuperación de los bosques de Gorgona. Consecuentemente, la comunidad de murciélagos debería ser considerado como uno de los objetos de conservación del PNN Gorgona.

\section{AGRADECIMIENTOS}

Este trabajo se realizó por solicitud del PNN Gorgona, en el marco del proyecto de investigación "Evaluación del estado actual de los objetos de conservación faunísticos en Isla Gorgona: una aproximación holística a la valoración ecológica del PNN Gorgona-CI7820", cofinanciado por el Fondo para la Acción Ambiental y la Niñez, Conservación Internacional Colombia, Universidad del Valle y Fundación Squalus; y amparado por el permiso de investigación científica PIBD-DTSO 011-10 del Ministerio del Medio Ambiente. Agradecemos a: Sergio Solari, Vladimir Rojas y Nathaly Calvo por sus aclaraciones taxonómicas acerca de los especímenes colectados en la isla; Jorge Velandia, Ana Paola Yusti, Manuel Sánchez, Lina Marcela Ortiz, Sebastián Orjuela, Adriana Chávez y Natalia Cuellar por la asistencia en el trabajo de campo; a los curadores de las colecciones de mamíferos del Instituto de Ciencias Naturales (ICN) y del Instituto de Investigación Alexander Von Humboldt (IAvH) por permitir el acceso a la información de las colecciones; a Ximena Zorrilla, Luis Payán, Héctor González y demás funcionarios del PNN Gorgona. Finalmente, agradecemos a tres evaluadores por sus importantes comentarios y sugerencias que contribuyeron a mejorar la calidad del manuscrito.

\section{RESUMEN}

Se cuantificó la variación de la comunidad de murciélagos en bosques con diferente grado de intervención en el
Parque Nacional Natural Gorgona. Para esto se realizaron capturas en tres zonas (dos sitios en cada zona) durante tres eventos de muestreo. El análisis de datos consideró la detección imperfecta, permitiendo realizar una inferencia confiable acerca de los cambios en presencia, abundancia y riqueza entre tipos de coberturas. Los resultados evidenciaron una considerable variación en detectabilidad relacionada con variaciones entre gremios tróficos y tipos de cobertura. Las tasas de presencia aumentaron al disminuir la cobertura boscosa $\left(\Psi_{\text {b.intervenido }}>\Psi_{\text {b. secundario }}>\Psi_{\text {b. primario }}\right)$ y fueron diferentes entre especies. La abundancia incrementó al aumentar el nivel de intervención $\left(N_{\mathrm{b} \text {.intervenido }}>N_{\mathrm{b}}\right.$. secundario $>N_{\mathrm{b} \text { primario }}$ ), disminuyó con el tamaño corporal y fue mayor para frugívoros y nectarívoros que para insectívoros. Los resultados sugieren que las comunidades de murciélagos despliegan fuertes respuestas al grado de intervención. Aunque se presentó mayor presencia y abundancia en el área más intervenida, los murciélagos también utilizaron las menos intervenidas. Esto puede incrementar el movimiento de las semillas entre diferentes estadíos sucesionales. Por lo tanto, el estudio de la comunidad de murciélagos puede ser importante para monitorear el estado de recuperación de los bosques del PNN Gorgona y para entender los procesos ecológicos que generan la recuperación de estos.

Palabras clave: Probabilidad de detección, probabilidad de presencia, gremios, tamaño corporal, riqueza de especies.

\section{REFERENCIAS}

Acevedo, C. I., Beltrán, B. S. \& Caicedo, R. A. (2004). Plan básico de manejo 2005-2009. Parque Nacional Natural Gorgona, Colombia.

Avila-Cabadilla, L. D., Stoner, K. E., Henry, M. \& AlvarezAñorve, M. Y. (2009). Composition, structure and diversity of phyllostomid bat assemblages in different successional stages of a tropical dry forest. Forest Ecology and Management, 258: 986-996.

Brosset, A., Charles-Dominique, P., Cockle, A., Cosson, J. F. \& Masson, D. (1996). Bat communities and deforestation in French Guiana. Canadian Journal of Zoology, 74: 1974-1982.

Brown, J. H., Gillooly, J. F., Allen, A. P., Savage, V. M. \& West, G. B. (2004). Toward a metabolic theory of ecology. Ecology, 85: 1771-1789.

Burnham, K. P. \& Anderson, D. R. (2002). Model selection and multimodel inference: a practical informationtheoretic approach. Springer-Verlag, New York, USA.

Burnham, K. P. (2004). Multimodel Inference: Understanding AIC and BIC in Model Selection. Sociological Methods \& Research, 33: 261-304.

Carrera, J. P. (2003). Distribución de murciélagos (Chiroptera) a través de un gradiente altitudinal en las estribaciones orientales de los Andes ecuatorianos 
(Tesis de pregrado). Pontificia Universidad Católica del Ecuador, Quito, Ecuador.

Castro-Luna, A. A., Sosa, V. J. \& Castillo-Campos, G. (2007). Bat diversity and abundance associated with the degree of secondary succession in a tropical forest mosaic in south-eastern Mexico. Animal Conservation, 10: 219-228.

Chao, A., Chazdon, R. L., Colwell, R. K. \& Shen, T. (2006). Abundance-based similarity indices and thir estimation when there are unknowned species in samples. Biometrics, 62: 361-371.

Chao, A., Colwell, R. K., Lin, C. W. \& Gotelli, N. J. (2009). Sufficient sampling for asymptotic minimum species richness estimators. Ecology, 90: 1125-1133.

Clarke, F. M., Rostant, L. V. \& Racey, P. A. (2005). Life after logging : post-logging recovery of a neotropical bat community. Journal of Applied Ecology, 42: 409-420.

Cleveland, C. J., Betke, M., Federico, P., Frank, J. D., Hallam, T. G., Horn, J., López, J. D. Jr., Mccracken, G. F., Medellín, R. A., Moreno-Valdez, A., Sansone, C. G., Westbrook, J. K. \& Kunz, T. H. (2006). Economic value of the pest control service provided by Brazilian free-tailed bats in south-central Texas. Front. Journal of Environment and Ecology, 4: 238-243.

Cuevas-Correa, D. (2007). Estimación de la densidad poblacional, patrón de distribución espacial y preferencia de hábitat del perezoso de tres dedos Bradypus variegatus gorgon y consideraciones para su conservación en el Parque Nacional Natural Gorgona (Tesis de pregrado). Pontificia Universidad Javeriana, Bogotá, Colombia.

Emmons, L. H. \& Feer, F. (1997). Neotropical rainforest mammals: a field guide. University of Chicago Press, Chicago, USA.

Estrada, A. \& Coates-Estrada, R. (2001). Species composition and reproductive phenology of bats in a tropical landscape at Los Tuxtlas, Mexico. Journal of Tropical Ecology, 17: 627-646.

Fenton, M. B., Acharya, L., Audet, D., Hickey, M. B. C., Merriman, C., Obrist, M. K., Syme, D. M. \& Adkins, B. (1992). Phyllostomid bats (Chiroptera: Phyllostomidae) as indicators of habitat disruption in the Neotropics. Biotropica, 24: 440-446.

Fiske, I. J. \& Chandler, R. B. (2011). unmarked : An R Package for Fitting Hierarchical Models of Wildlife Occurrence and Abundance. Journal of Statistical Software, 43: 1-23.

Fleming, T. H. \& Heithaus, E. R. (1981). Frugivorous bats, seed shadows, and the structure of tropical forests. Biotropica, 13: 45-53.

Fleming, T. H. \& Williams, C. F. (1990). Phenology, seed dispersal, and recruitment in Cecropia peltata
(Moraceae) in Costa Rican tropical dry forest. Journal of Tropical Ecology, 6: 163-178.

Flemming, T. (1992). Interactions within herbivore communities mediated by the host plants: The keystone herbivore concept. In T. Hunter, T. Ohgushi \& P. W. Price (Eds.), Effects of Resource Distribution on Animal Plant Interactions (pp. 287-325). San Diego, California, USA

Galindo-González, J. (1998). Dispersión de semillas por murciélagos: su importancia en la conservación y regeneración del bosque tropical. Acta Zoológica Mexicana, 73: 57-74.

García-Morales, R., Badano, E. I. \& Moreno, C. E. (2013). Response of Neotropical Bat Assemblages to Human Land Use. Conservation Biology, 27: 1-11.

Gorchov, D. L., Cornejo, F., Ascorra, C. \& Jaramillo, M. (1993). The role of seed dispersal in the natural regeneration of rain forest after strip-cutting in the Peruvian Amazon. Vegetatio, 107: 339-349.

Gotelli, N. J. \& Colwell, R. K. (2001). Quantifying biodiversity: procedures and pitfalls in the measurement and comparison of species richness. Ecology Letters, 4: $379-391$

Gotelli, N. J. \& Colwell, R. K. (2011). Estimating species richness. In A. E. Magurran \& B. J. McGill (Eds.), Biological Diversity (pp. 39-54). Oxford University Press, New York, USA.

Hernández-Conrique, D., Iniguez-Davalos. L. I. \& Storz, J. F. (1997). Selective Feeding by Phyllostomid Fruit Bats in a Subtropical Montane cloud forest. Biotropica, 29: 376-379.

Karr, J. R. (1976). Seasonality, resource availability and community diversity in tropical bird community. American Naturalist, 110: 973-994.

Karr, J. R. (1981). Surveying birds with mist nets. In C. J. Ralph \& J. M. Scott (Eds.), Estimating Numbers of Terrestrial Birds (pp. 62-67). Studies in Avian Biology. The Blackburn Press, Caldwell, New Jersey, USA.

Karr, J. R. (1990). The avifauna of Barro Colorado Island and the Pipeline Road, Panama. In A. H. Gentry (Ed.), Four Neotropical Forests (pp. 183-198). Yale University Press, Connecticut, USA.

Kerr, S. R. \& Dickie, L. M. (2001). The biomass spectrum : a predator-prey theory of aquatic production, Complexity in Ecological Systems. Columbia University Press, New York, USA.

Law, B. S., Anderson, J. \& Chidel, M. (1999). Bat communities in a fragmented forest landscape on the south-west slopes of New South Wales, Australia. Biological Conservation, 88: 333-345.

Lim, B. K. \& Engstrom, M. D. (2001). Species diversity of bats ( Mammalia: Chiroptera ) in Iwokrama Forest, Guyana, and the Guianan subregion: implications 
for conservation. Biodiversity and Conservation, 10 613-657.

Loayza, A. P., Rios, R. S. \& Larrea-Alcázar, D. M. (2006). Disponibilidad de recurso y dieta de murciélagos frugívoros en la Estación Biológica Tunquini, Bolivia. Ecología en Bolivia, 41: 7-23.

Lobova, T. A., Mori, S. A., Blanchard, F., Peckham, H. \& Charles-Dominique, P. (2003). Cecropia as a food resource for bats in French Guiana and the significance of fruit structure in seed dispersal and longevity. American Journal of Botany, 90: 388-403.

Longcore, T. \& Rich, C. (2004). Ecological light pollution. Frontiers in Ecology and the Environment, 2: 191-198.

MacKenzie, D. I., Nichols, J. D., Lachman, G. B., Droege, S., Andrew-Royle, J. \& Langtimm, C. A. (2002). Estimating site occupancy rates when detection probabilities are less than one. Ecology, 83: 2248-2255.

MacKenzie, D., Nichols, J. \& Hines, J. (2003). Estimating site occupancy, colonization, and local extinction when a species is detected imperfectly. Ecology, 84: 2200-2207.

MacKenzie, D. I., Nichols, J. D., Royle, J. A., Pollock, K. H., Bailey, L. L. \& Hines, J. E. (2006). Occupancy estimation and modeling: inferring patterns and dynamics of species occurrence. Academic Press, San Diego, California, USA.

Martin, T. E. \& Karr, J. R. (1986). Temporal dynamics of neotropical birds with special reference to frugivores in second-growth woods. Wilson Bulletin, 98: 38-60.

Medellín, R. A., Equihua, M. \& Amin, M. A. (2000). Bat diversity and abundance as indicators of disturbance in Neotropical rainforests. Conservation Biology, 14: 1666-1675

Meyer, C. F. J., Aguiar, L. M. S., Aguirre, L. F., Baumgarten, J., Clarke, F. M., Cosson, J. F., Villegas, S. E., Fahr, J., Faria, D., Furey, N., Henry, M., Hodgkison, R., Jenkins, R. K. B., Jung, K. G., Kingston, T., Kunz, T. H., MacSwiney-Gonzalez, M. C., Moya, I., Patterson, B. D., Pons, J. M., Racey, P. A., Rex, K., Sampaio, E. M., Solari, S., Stoner, K. E., Voigt, C. C., von Staden, D., Weise, C. D. \& Kalko, E. K. V. (2011). Accounting for detectability improves estimates of species richness in tropical bat surveys. Journal of Applied Ecology, 48: 777-787.

Mooney, H. A., Lubchenco, J., Dirzo, R. \& Sala, O. E. (1995). Biodiversity and ecosystem functioning: basic principles. In V. H. Heywood (Ed.), Global Biodiversity Assessment (pp. 275-325). Cambridge University Press, Cambridge, United Kingdom.

Muñoz, J. (2001). Los murciélagos de Colombia: sistemática, distribución, descripción, historia natural y ecología. Universidad de Antioquia, Medellín, Colombia.
Noss, R. F. (1990). Indicators for Monitoring Biodiversity: A Hierarchical Approach. Conservation Biology, 4: 355-364.

Passos, F. C., Silva, W. R., Pedro, W. A. \& Bonin, M. R. (2003). Frugivoria em morcegos (Mammalia, Chiroptera) no Parque Estadual Intervales, sudeste do Brasil. Revista Brasileira de Zoologia, 20: 511-517.

Patterson, B. D., Willig, M. R. \& Stevens, R. D. (2003). Trophic strategies, niche partitioning, and patterns of ecological organization. In T. H. Kunz \& M. B. Fenton (Eds.), Bat Ecology (pp. 536-579). University of Chicago Press, Chicago, USA.

Pierson, E. D. (1998). Tall trees, deep holes, and scarred landscapes: conservation biology of North American bats. In T. H. Kunz (Ed.), Bat Biology and Conservation (pp. 309-325). Smithsonian Inst Scholarly Press, Washington, USA.

Pyke, G. H. (1983). Seasonal pattern of abundance of honeyeaters and their resources in heathland areas near Sydney. Australian Journal of Ecology, 8: 217-233.

R Development Core Team. (2012). R: A language and environment for statistical computing. R Foundation for Statistical Computing, Vienna, Austria.

Royle, J. A. \& Dorazio, R. M. (2006). Hierarchical models of animal abundance and occurrence. Journal of Agricultural, Biological, and Environmental Statistics, 11: 249-263.

Royle, J., Kéry, M., Gautier, R. \& Schmid, H. (2007). Hierarchical spatial models of abundance and occurrence from imperfect survey data. Ecological Monographs, 77: 465-481.

Royle, J. A. \& Dorazio, R. M. (2008). Hierarchical modeling and inference in ecology: the analysis of data from populations, metapopulations and communities. Academic Press. San Diego, California, USA.

Rydell, J. \& Baagøe, H. J. (1996). Bats and streetlamps. Bat Conservation International, 14: 10-13.

Schulze, M. D., Seavy, N. E. \& Whitacre, D. F. (2000). A comparison of the phyllostomid bat assamblages in undisturbed neotropial forest and in forest fragments of a slash-and-burn farming mosaic in Petén, Guatemala. Biotropica, 32: 174-184.

Simmons, N. B. \& Voss, R. S. (2001). The mammals of Paracou, French Guiana: A neotropical lowland rainforest fauna-part 1, Bats. Bulletin of the American Museum of Natural History, 237: 1-219.

Soriano, P. J. (2000). Functional structure of bat communities in tropical rainforests and Andean cloud forests. Ecotropicos, 13: 1-20.

Tirira, D. (2008). Mamíferos de los bosques húmedos del noroccidente de Ecuador. Ediciones murciélago blanco, Quito, Ecuador. 
Torres, E. (1986). Historia de Gorgona. In H. Von Prahl \& M. Alberico (Eds.). Isla de Gorgona (pp. 9-17). Biblioteca textos universitarios, Bogotá, Colombia.

Voss, R. S. \& Emmons, L. H. (1996). Mammalian diversity in neotropical lowland rainforests : a preliminary assessment. Bulletin of the American Museum of Natural History, New York, USA.

White, E. P., Ernest, S. K. M., Kerkhoff, A. J. \& Enquist, B. J. (2007). Relationships between body size and abundance in ecology. Trends in Ecology \& Evolution, 22: 323-330.
Williams, B. K., Nichols, J. D. \& Conroy, M. J. (2002). Analysis and management of animal populations. Academic Press, San Diego, California, USA.

Willig, M. R., Presley, S. J., Bloch, C. P., Hice, C. L., Yanoviak, S. P., Díaz, M. M., Chauca, L. A., Pacheco, V. \& Weaver, S. C. (2007). Phyllostomid Bats of Lowland Amazonia: Effects of Habitat Alteration on Abundance. Biotropica, 39: 737-746.

Woodward, G., Ebenman, B., Emmerson, M., Montoya, J. M., Olesen, J. M., Valido, A. \& Warren, P. H. (2005). Body size in ecological networks. Trends in Ecology \& Evolution, 20: 402-409. 